\title{
Efficacy of lenvatinib in a patient with anaplastic thyroid cancer
}

\author{
Kazuyuki Oishi, Daisuke Takabatake and Yuichi Shibuya \\ Kochi Health Sciences Center - Divisions of Breast \& Thyroid Surgery, Kochi, Japan
}

\section{Summary}

We experienced a case of an 82-year-old woman who presented to our hospital with a 1-month history of dysphagia and dyspnea. Cervical contrast-enhanced computed tomography revealed diffuse thyroid neoplasms causing significant tracheal stenosis with tumors, particularly of the superior mediastinum, which were associated with an embolism of the brachiocephalic vein and suspected invasion to the bilateral common carotid arteries. Anaplastic thyroid cancer (ATC) was diagnosed by fine-needle aspiration; thus, emergency tracheostomy and gastrostomy were performed. We made a definitive diagnosis of ATC (T4bNOMO Stage IVB) and initiated continuous lenvatinib administration at $24 \mathrm{mg} /$ day. Although several adverse events occurred, the tumor size reduced remarkably over a short period. However, the patient died from rupture of the common carotid artery 30 days after treatment initiation. Here, we report our experience with lenvatinib therapy for ATC and include a literature review.

\section{Learning points:}

- Lenvatinib is extremely effective for ATC.

- Lenvatinib has a much greater cytoreductive effect than traditional therapies, but it needs dose reduction or withdrawal because of treatment-related side effects.

- Lenvatinib may cause treatment-related carotid blowout syndrome, resulting in death for patients with invasion to the carotid artery.

\section{Background}

Most patients with anaplastic thyroid cancer (ATC) have a bulky neck tumor with cervicodynia, dyspnea, dysphagia, hoarseness and cough. More than 90\% of patients have local infiltration to the great vessels around the trachea, esophagus and mediastinum. Distant metastasis occurs in $15-50 \%$ of patients (1). The disease-specific mortality is approximately $100 \%$, the median survival time is 5 months and the 1-year survival rate of $20 \%$ is extremely poor (2). There have been few reported cases of resectable ATC in which a tumor is contained within the thyroid gland. Combined modality therapy is often used. Combined use of docetaxel, cisplatin, radiation and doxorubicin has been reported; however, the treatment led to poor outcomes; therefore, established treatments do not exist (3).

Recently, molecular-targeted drugs have become available, and the efficacy of ATC treatment has been expected to rise. The efficacy of lenvatinib to treat radioiodine-refractory $\mathrm{TC}$ was demonstrated in the SELECT study; however, the subjects had differentiated TC and poorly differentiated TC only (4). The 208 trials, which investigated the efficacy of lenvatinib to treat unresectable medullary TC and ATC along with radioiodine-refractory TC, demonstrated good results in Japan (5). Seventeen patients with ATC were enrolled. The median progressionfree survival was 7.4 months, and the median overall 
survival was 10.6 months. The objective response rate was $24 \%$ ( 4 cases); the disease control rate was $94 \%$ (16 cases) out of 17 total cases (complete response: 0 cases; partial response (PR): 4 cases; stable disease: 12 cases; and progressive disease, 1 case). There have not been any other results to which these results could be compared, and further studies need to be conducted.

In this case study, a short-term cytoreductive effect of lenvatinib on ATC was obtained; however, lenvatinib caused treatment-related carotid blowout syndrome (CBS), which resulted in death. We present this case of ATC as a reference that may provide guidance on lenvatinib treatment for future ATC cases.

\section{Case presentation}

The patient was an 82-year-old woman. She had a rapidly increasing neck tumor, dysphagia and dyspnea in May 2015 and consulted a medical institution in June where she had been diagnosed with a thyroid tumor. She was referred to the Kochi Health Sciences Center for detailed examinations and therapy because computed tomography (CT) revealed a diffuse large tumor around the trachea, and the examination of the larynx fiber revealed bilateral vocal cord paresis. The anamnesis revealed that the patient had high blood pressure and had taken amlodipine, but there was no specific description of her family history. Blood tests revealed that her white blood cell count had increased to $21270 / \mu \mathrm{L}$; she had subclinical hypothyroidism (FT3, 2.40 pg/mL; FT4, $1.00 \mathrm{ng} / \mathrm{dL}$; and thyroid-stimulating hormone (TSH), $6.747 \mu \mathrm{IU} / \mathrm{mL}$ ), and her antibody levels had all increased (Tg, $47.3 \mathrm{ng} / \mathrm{mL}$; TgAb, $470 \mathrm{IU} / \mathrm{mL}$; and TPOAb, $432 \mathrm{IU} /$ $\mathrm{mL})$. Neck ultrasound examination revealed a diffuse, large tumor with an irregular shape, indistinct border, low echoic nature and coarse calcification. ATC was strongly suspected. There was no clear invasion into the trachea, as viewed using a bronchoscope, or into the esophagus, as viewed using an esophagus endoscope; however, tracheal and esophageal stenosis caused by tumor compression from the outside was observed. The thyroid tumor was buried in the superior mediastinum and had caused tracheal stenosis, invasion into the bilateral common carotid arteries and a tumor embolism in the right brachiocephalic vein, as determined by contrast-enhanced CT (Fig. 1A, B and C). Positron emission tomography revealed [18F]-fluorodeoxyglucose accumulation in the thyroid tumor (Fig. 1D). We admitted her to the hospital on the first day of examination and performed aspiration
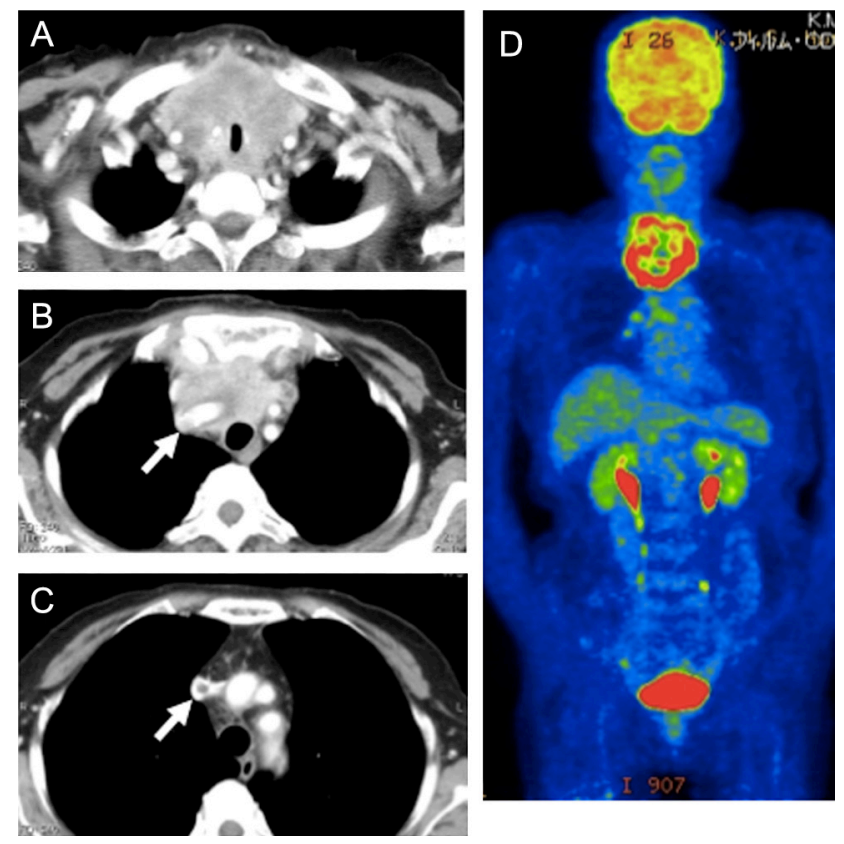

Figure 1

Cervical contrast-enhanced $\mathrm{CT}$ findings showing a diffuse thyroid tumor extending to the mediastinum, infiltrating both the common carotid arteries and pressing the trachea from the bilateral side with stenosis (A). Tumor thrombosis is observed from the right brachiocephalic vein to the superior vena cava (arrows) (B, C). Positron emission tomographic examination showing the accumulation of [18F]-fluorodeoxyglucose in the diffuse thyroid tumor, but there is lack of accumulation inside the tumor because of necrosis (D).

biopsy cytology; she was diagnosed with ATC (T4bNOMO Stage IVB).

Her stridor had worsened the next day; thus, we performed a tracheostomy to treat the tracheal stenosis and a gastrostomy to treat esophageal stenosis (Fig. 2). Considering that we recognized a tendency toward rapid tumor enlargement in the short term, weekly administration of paclitaxel with radiation therapy or lenvatinib therapy was considered as a treatment strategies. We did not choose chemoradiotherapy because wound
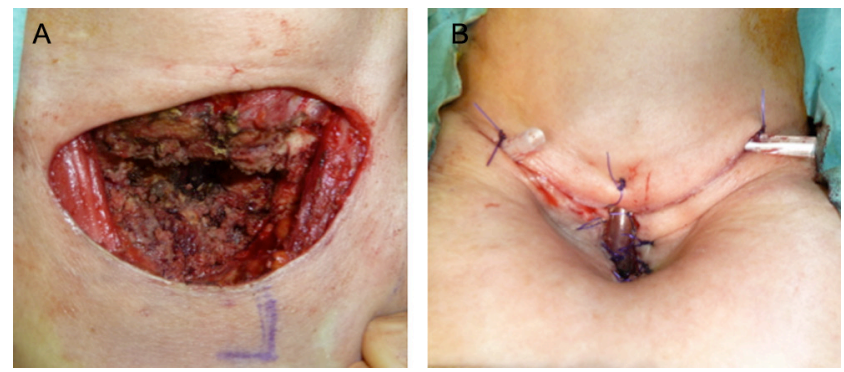

Figure 2

The partially excised tumor is shown under an approximately 10-cm collar incision (A). A view of the tracheostomy is shown (B). 
healing could be delayed due to radiation exposure just after the tracheostomy. Although delayed wound healing is a well-known side effect related to vascular endothelial growth factor receptor inhibitor administration, we considered the benefit of lenvatinib to be better than its side effect at that time and chose to initiate lenvatinib therapy. We initiated lenvatinib administration at dose $24 \mathrm{mg}$ once a day on day 5 after hospitalization. Lenvatinib was delivered through a gastric tube because it was difficult for the patient to normally ingest the medication.

The response was objectively evaluated by medical imaging findings and the Response Evaluation Criteria in Solid Tumors. Adverse events were evaluated using the Common Terminology Criteria for Adverse Events (version 4.0).

\section{Outcome and follow-up}

There was an obvious rapid reduction in the tumor size following lenvatinib administration each day. The effect was judged to be PR by contrast-enhanced CT on day 8 after lenvatinib administration compared with the disease status on the first day; however, there was no change in the tumor embolism, and further reduction was observed on day 29 (PR) (Fig. 3). There was no improvement in swallowing and salivary leakage around the site of tracheostomy, and the patient continued to need sustained suction; however, her dyspnea dramatically improved, and her ability to get out of bed and her performance status remained between 1 and 2 .

We accepted Grade 3 hypertension, Grade 2 venous thrombosis, Grade 2 proteinuria, Grade 1 diarrhea and
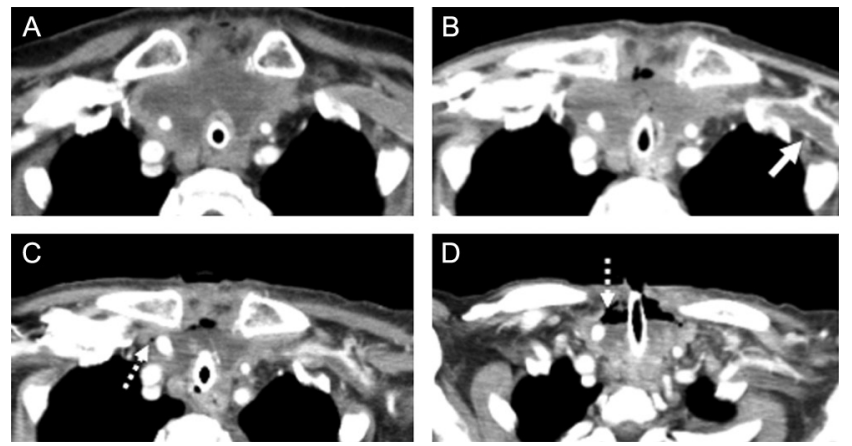

Figure 3

The change in cervical contrast-enhanced CT findings after lenvatinib administration. The tumor occupied the superior mediastinum on the $1 \mathrm{st}$ day (A). The tumor was moderately reduced, but air appeared in the ventral aspect of the tumor, and thrombosis was observed in the left axillary vein on the 8th day (solid line) (B). The tumor was further reduced (partial response), but air had spread around the right common carotid artery by the 29th day (dotted line) (C and D). fatigue as adverse events. On day 22, we initiated LT4 (levo-thyroxine) administration at $25 \mu \mathrm{g} /$ day, which was increased to $50 \mu \mathrm{g} /$ day on day 29 because of increasing TSH. Furthermore, we administered angiotensin receptor II blocker for high blood pressure. We discontinued lenvatinib on days 24-29 and resumed administration with a reduced dose of $20 \mathrm{mg}$ on day 30 because of the appearance of a side effect of more than Grade 2, such as hypertension, venous thrombosis and proteinuria; however, the patient died from massive bleeding from the right common artery.

\section{Discussion}

Lenvatinib is an oral, multi-targeted, tyrosine kinase inhibitor of the vascular endothelial growth factor receptors 1, 2 and 3; fibroblast growth factor receptors 1-4; platelet-derived growth factor receptor alpha; and the RET and KIT signaling pathways $(6,7)$. The reported adverse events include hypertension, diarrhea, fatigue, decreased appetite, decreased weight, nausea, stomatitis, palmarplantar erythrodysesthesia syndrome, proteinuria and vomiting. Prompt intervention is particularly required for hypertension because of the rapid deterioration of the patient after the administration of lenvatinib for a few days (4). In our case, we experienced CBS, which has not been reported to date. To the best of our knowledge, this is the first case reported to examine lenvatinib activity in a patient with ATC and to demonstrate carotid common artery rupture as an adverse event.

We observed the foramen of the ruptured right common carotid artery, and pathological analysis of a specimen obtained from the site revealed a strong inflammatory cell permeation and partial necrosis (Fig. 4). The tumor itself, which invaded the common carotid artery, was necrotized by the effect of lenvatinib, and an artery wall had become weak and was near failure.
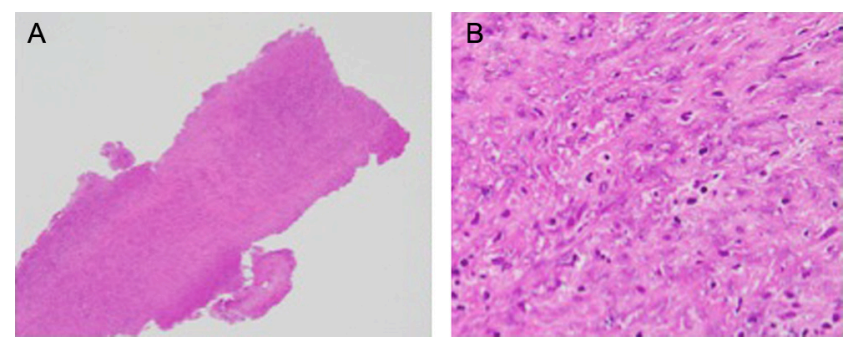

Figure 4

Histopathological examination of the ruptured artery showing strong permeation of inflammatory cells and partial necrosis of the arterial wall. (A) H\&E stain $\times 4$ and (B) H\&E stain $\times 40$. 
It was very likely that the infection caused by saliva that was aspirated and flowed into the tracheostomy area had promoted delayed wound healing, tissue necrosis and blood vessel permeation around the common carotid artery. Contrast-enhanced CT performed on the day before bleeding revealed that the common carotid artery was exposed to the necrotic part of the tumor; therefore, we may have been able to predict the occurrence of bleeding from these observations. Lenvatinib is extremely effective for ATC, but when the invasion of the neighboring great vessels in particular is suspected, we confirm the antitumor effects by assessing the adverse events, performing physical examinations, and studying images, such as those from CT, and appropriate reduction and withdrawal of antitumor effects are essential measures of efficacy. Discontinuation of treatment with lenvatinib will help cover the peritumoral dead space by allowing the tumor to re-grow, thereby preventing the occurrence of CBS in patients with ATC, and resumption of the treatment will reduce tumor size; this cycle is continued for an appropriate period of time, and even if the dose of lenvatinib is small, continuation of administration is recommended for as long as possible. In this case, the speed of shrinking was extremely high; therefore, to confirm the change in the tumor size, only CT that is performed every 2 or 3 weeks can objectively evaluate treatment progress. However, because of the increased radiation exposure from frequent CT scans, the evaluation method should be reviewed from the standpoint of the patient's safety.

To date, we have used combined therapy, such as chemotherapy and radiotherapy, to treat ATC; however, the benefits have appeared to be limited. We expected improved efficacy for lenvatinib and used it for ATC in this case. We observed a much greater cytoreductive effect than expected, and the effect may have been extremely strong; therefore, the dose of $24 \mathrm{mg}$ /day might be extremely high for Japanese patients. However, we have no evidence suggesting that doses $<24 \mathrm{mg}$ /day are efficacious; hence, we continue to administer $24 \mathrm{mg}$ /day at present.

Although the administration of molecular-targeted drugs just after tracheostomy was considered to be a special situation for ATC in this case, it is not normal. Delayed wound healing following treatment with lenvatinib has been reported to be an adverse event because of its anti-angiogenic effect, and discontinuation before surgical management has been recommended, but this recommendation was based only on the assessment of the general status of patients after surgical management.
We should have investigated methods with various delays in initiating administration after tracheostomy or methods involving dose withdrawal.

CBS is a life-threatening complication of head and neck cancer, and infection complicates its management. Traditional surgical ligation is effective for preventing hemorrhage but frequently leads to a high risk of cerebral complications. Currently, endovascular embolization and stent placement have become the dominant therapeutic strategies $(8,9)$. Although the long-term safety, stent patency and hemostasis permanency appear to be unfavorable, it is the only useful method for patients with ATC who are close to developing CBS. Further research is required in the areas of tracheostomy and parenteral administration, adaptation, dose and route of administration of lenvatinib in typical cases of ATC, such as large vascular invasion.

The occurrence of ATC is extremely rare; thus, conducting large-scale studies in a single institution is impossible. Therefore, it would be useful for institutions to cooperate in sharing information, accumulating cases and developing appropriate protocols. We expect that lenvatinib will become an effective therapy for ATC, for which there is no established therapy currently.

\section{Declaration of interest}

The authors declare that there is no conflict of interest that could be perceived as prejudicing the impartiality of the research reported.

\section{Funding}

This research did not receive any specific grant from any funding agency in the public, commercial or not-for-profit sector.

Author contribution statement

Kazuyuki Oishi is the first author and contact author and was responsible for the writing the case.

\section{References}

1 McIver B, Hay ID, Giuffrida DF, Dvorak CE, Grant CS, Thompson GB, van Heerden JA \& Goellner JR 2001 Anaplastic thyroid carcinoma: a 50-year experience at a single institution. Surgery 130 1028-1034. (doi:10.1067/msy.2001.118266)

2 Smallridge RC \& Copland JA 2010 Anaplastic thyroid carcinoma: pathogenesis and emerging therapies. Clinical Oncology 22 486-497. (doi:10.1016/j.clon.2010.03.013)

3 Foote RL, Molina JR, Kasperbauer JL, Lloyd RV, McIver B, Morris JC, Grant CS, Thompson GB, Richards ML, Hay ID, et al. 2011 Enhanced survival in locoregionally confined anaplastic thyroid carcinoma: a single-institution experience using aggressive multimodal therapy. Thyroid 21 25-30. (doi:10.1089/thy.2010.0220) 
4 Schlumberger M, Tahara M, Wirth LJ, Robinson B, Brose MS, Elisei R, Habra MA, Newbold K, Shah MH, Hoff AO, et al. 2015 Lenvatinib versus placebo in radioiodine-refractory thyroid cancer. New England Journal of Medicine 372 621-630. (doi:10.1056/NEJMoa1406470)

5 Tahara M, Kiyota N, Yamazaki T, Chayahara N, Nakano K, Inagaki L, Toda K, Enokida T, Minami H, Imamura Y, et al. 2017 Lenvatinib for anaplastic thyroid cancer. Fronttiers in Oncology 7 25. (doi:10.3389/fonc.2017.00025)

6 Matsui J, Funahashi Y, Uenaka T, Watanabe T, Tsuruoka A \& Asada M 2008 Multi-kinase inhibitor E7080 suppresses lymph node and lung metastases of human mammary breast tumor MDA-MB-231 via inhibition of vascular endothelial growth factor-receptor (VEGF-R) 2 and VEGF-R3 kinase. Clinical Cancer Research 14 5459-5465. (doi:10.1158/1078-0432.CCR-07-5270)
7 Matsui J, Yamamoto Y, Funahashi Y, Tsuruoka A, Watanabe T, Uenaka T \& Asada M 2008 E7080, a novel inhibitor that targets multiple kinases, has potent antitumor activities against stem cell factor producing human small cell lung cancer H146, based on angiogenesis inhibition. International Journal of Cancer 122 664-671. (doi:10.1002/ijc.23131)

8 Patsalides A, Fraser JF, Smith MJ, Kraus D, Gobin YP \& Riina HA 2010 Endovascular treatment of carotid blowout syndrome: who and how to treat. Journal of Neurointerventional Surgery 2 87-93. (doi:10.1136/ jnis.2009.001131)

9 Warren FM, Cohen JI, Nesbit GM, Barnwell SL, Wax MK \& Andersen PE 2002 Management of carotid 'blowout' with endovascular stent grafts. Laryngoscope 112 428-433. (doi:10.1097/00005537-20020300000004)

Received in final form 20 April 2017

Accepted 4 May 2017 